\title{
Treatment response in relation to inflammatory and axonal surrogate marker in multiple sclerosis
}

\author{
A. Petzold ${ }^{\mathrm{a}, \mathrm{d}, *}$, D. Brassat b,d, P. Mas ${ }^{\mathrm{b}}$, K. Rejdak ${ }^{\mathrm{a}, \mathrm{c}}$, \\ G. Keir ${ }^{\text {a }}$, G. Giovannoni ${ }^{\text {a }}$, EJ. Thompson ${ }^{\mathrm{a}}$, M. Clanet ${ }^{\mathrm{b}}$, \\ ${ }^{\text {a } U C L ~ I n s t i t u t e ~ o f ~ N e u r o l o g y, ~ D e p a r t m e n t ~ o f ~ N e u r o i m m u n o l o g y, ~ Q u e e n ~ S q u a r e, ~}$ \\ London WC1N 3BG, United Kingdom \\ ${ }^{\mathrm{b}}$ Department of Neurology, Centre Hospitalier Purpan, Toulouse, France \\ ${ }^{\mathrm{c}}$ Department of Neurology, Medical University, Lublin, Poland \\ ${ }^{\mathrm{d}}$ both authors contributed equally to the study
}




\section{Abstract}

Background: This study aimed to investigate if treatment response could retrospectively be related to inflammatory or axonal pathology as measured by plasma surrogate markers.

Methods: In this 1-year observational study 30 MS patients with relapsingremitting disease were treated with intra-muscular IFN $\beta-1$ a or subcutaneous IFN $\beta$ 1b. Responders and non-responders were defined according to clinical and MRI criteria. The control group consisted of 14 healthy subjects. Plasma levels of surrogate markers for inflammation (nitric oxide metabolites $\left(\mathrm{NO}_{x}\right)$ ), astrocytic activation (S100B) and axonal damage ( $\mathrm{NfH}^{S M I 35}$ ) were measured using standard assays.

Results: There were 11 non-responders and 19 responders to IFN $\beta$ treatment. Median S100B levels were elevated in a higher proportion of treatment responders $(63 \%, 42.9 \mathrm{pg} / \mathrm{mL})$ compared to non-responders $(18 \%, 11.7 \mathrm{pg} / \mathrm{mL}, \mathrm{p}<0.05$, Fisher's exact test) and controls $(0 \%, 2 \mathrm{pg} / \mathrm{mL}, \mathrm{p}<0.001)$. Levels of $\mathrm{NO}_{x}$ were found to be more frequently elevated in non-responders $(72 \%, 39 \mu \mathrm{M})$ compared to healthy controls $(0 \%, 37 \mu \mathrm{M}, \mathrm{p}<0.05)$. Levels of $\mathrm{NfH}^{S M I 35}$ were more frequently elevated in responders $(58 \%, 300 \mathrm{pg} / \mathrm{mL}, \mathrm{p}<0.001)$ and non-responders $(72 \%, 500 \mathrm{pg} / \mathrm{mL}$, $\mathrm{p}<0.001)$ compared to controls $(0 \%, 4.5 \mathrm{pg} / \mathrm{mL})$.

Conclusion: Patients with relapsing-remitting MS who had surrogate marker supported evidence for astrocytic activation responded more frequently to treatment with $\operatorname{IFN} \beta$.

Key words: $\mathrm{S} 100 \mathrm{~B}$, neurofilament, $\mathrm{NfH}^{S M I 35}, \mathrm{NO}_{x}$, surrogate marker, multiple sclerosis, treatment response

\footnotetext{
* Corresponding author. Tel. +44 (0)207 8373611 ext. 4204, Fax. +44 (0)207 837 8553

Email address: a.petzold@ion.ucl.ac.uk (A. Petzold ).
} 


\section{Introduction}

Characterising responders and non-responders to treatment in multiple sclerosis (MS) is of relevance for understanding disease progression and selecting effective drug treatment strategies. MS is a heterogeneous disease [1] and it was suggested that the heterogeneity of treatment response could be related to the underlying pathology [2]. Particularly inflammation has been associated with relapses, and relapse frequency early on is related to disease progression. The proven benefit common to all currently recommended treatments is a reduction in the rate of clinical relapse, which has been associated to a transient slowing of disease progression [3]. During the early phase of MS active disease has been related to elevated levels of S100B [4] and the nitric oxide metabolites nitrate and nitrite $\left(\mathrm{NO}_{x}\right)[5]$.

The hypothesis underlying this study was that patients who responded to IFN $\beta$ treatment would be different from non-responders with regard to their profile of plasma inflammatory $\left(\mathrm{NO}_{x}\right)$, astrocytic activation $(\mathrm{S} 100 \mathrm{~B})$ and axonal $\left(\mathrm{NfH}^{\text {SMI35 }}\right)$ markers.

\section{Materials and methods}

\subsection{Patients}

Thirty patients with relapsing remitting multiple sclerosis (RRMS) [6] were included in this cross-sectional study. All patients were treated with IFN $\beta$ and recruited from the MS specialist clinic at the Toulouse Purpan Hptial between March 2000 and November 2001. The control group consisted of 14 healthy volunteers. All plasma samples were obtained by antecubital venopuncture at the last clinical assessment. Samples were coded and stored at -70C until analysis. Approval for the study was obtained from the local Ethics Committees. Written informed consent was obtained from each subject.

\subsection{Clinical assessment}

All patients were assessed by two experienced neurologists (DB, MC). The expanded Disability Status Scale score (EDSS) and the yearly relapse frequency prior to sampling were recorded.

It was decided to declare treatment failure based on clinical and MRI criteria (when available). Patients who suffered from at least two clinical relapses as- 
sociated with at least one gadolinium-enhancing lesion on T1 MRI performed at least one month after steroid treatment ("active MRI"), or patients who progressed by over 1 point on the EDSS associated with an active MRI.

\subsection{Assays}

Plasma levels of $\mathrm{S} 100 \mathrm{~B}, \mathrm{NO}_{x}$ and $\mathrm{NfH}^{\text {SMI35 }}$ were measured using standard assays) as described previously $[7,8,9,10]$. The cutoff value for abnormal levels of these biomarkers were defined as the top value of the control group.

\subsection{Statistical Analysis}

All statistical analyses and graphs were done using SAS software (V8.2, SAS Institute, Inc., Cary, North Carolina, USA). Because of the non-Gaussian distribution all data is presented as the median and interquartile range (IQR). The box (median and 25\%-75\% cumulative frequency) and whisker (1\%-100\% cumulative frequency) are shown in the graphs. Because of the small sample size Fisher's exact test was used for comparison of proportions of patients. The linear relationship between continuous variables was evaluated using the non-parametric Spearman correlation coefficient $(\alpha=0.05)$.

\section{Results}

The baseline characteristic of the MS patients are summarised in Table 1. According to our definition there were 11 responder and 19 non-responder. Patients with MS were significantly younger than the control patients and there were more females. There were no significant differences between responder and non-responder with regard to the EDSS. The choice of the generic drug was not related to treatment response.

The plasma levels for $\mathrm{S} 100 \mathrm{~B}, \mathrm{NO}_{x}$ and $\mathrm{NfH}^{\text {SMI35 }}$ (median, range) are summarised in Table 1. The cutoff levels were determined as the top value from the control group $\left(\mathrm{S} 100 \mathrm{~B}=33 \mathrm{pg} / \mathrm{mL}, \mathrm{NO}_{x}=44 \mu \mathrm{M}, \mathrm{NfH}^{S M I 35}=250 \mathrm{pg} / \mathrm{mL}\right)$.

In all non-responders MRI was performed. Although a subgroup of responder $(\mathrm{n}=16)$ received an MRI brain scan. However the timing of MRI brain scan to relapse and plasma sampling was not rigorous.

A significantly higher proportion of responders (63\%) but not of non-responders (18\%) had S100B levels above cut-off compared to controls $(\mathrm{p}=0.021)$. The 
individual values are shown in Figure 1. There were 2 outliers with plasma S100B levels over $300 \mathrm{pg} / \mathrm{mL}$ among the responders (Figure 1). Removal of these 2 outliers did not alter statistical significance $(\mathrm{p}=0.034$, Fisher's exact test).

A significant proportion of non-responders (45\%) had $\mathrm{NO}_{x}$ levels above cutoff. Also a significant proportion of responders (58\%) and non-responders (72\%) had $\mathrm{NfH}^{\text {SMI35 }}$ levels above cutoff. There was no significant difference comparing proportions of responders and non-responders for either $\mathrm{NO}_{x}$ or $\mathrm{NfH}^{\text {SMI35 }}$.

There was no correlation between age, EDSS, relapse frequency and either $\mathrm{S} 100 \mathrm{~B}, \mathrm{NO}_{x}$ or $\mathrm{NfH}^{\mathrm{SMI} 35}$ for any of the subgroups.

\section{Discussion}

This study found higher plasma levels $\mathrm{S} 100 \mathrm{~B}, \mathrm{NO}_{x}$ and $\mathrm{NfH}^{S M I 35}$ in patients with MS than in healthy controls. In treatment responders the median S100B levels were over 3-fold higher than in non-responders.

We and others previously related elevated CSF S100B to acute disease activity in MS [4]. It is therefore difficult to understand if not paradoxic that treatment responders should have higher plasma S100B levels. A major criticism of this study is the retrospective, the cross-sectional design and the lack of MRI data in some of the patients. However in a longitudinal study on serum S100B in primary progressive MS patients from a clinically negative phase II trial we found similar levels in the non-responders (E Lim, unpublished data).

The current understanding is that IFN- $\beta$ reduces disease activity by exerting immunomodulatory properties. However in vivo and in vitro data shows upregulation of pro-inflammatory factors during IFN- $\beta$ treatment [11]. On the background that inflammation has been related to protective and destructive mechanisms [12] it can be speculated that these properties might be related in degree to a spectrum of severity. Severe inflammation, blood-brain barrier (BBB) disruption and neutrophil invasion were all related to tissue destruction [13]. Indeed there was a trend for higher $\mathrm{NfH}^{\text {SMI35 }}$ levels in non-responder. Previously we have shown that particular the combination of inflammation with axonal damage indicated poorer prognosis [14].

However low grade inflammation orchestrated by the macrophage and microglial network could be neuroprotective. In vitro data supports this notion in as nanomolar levels of S100B are neurotrophic [15]. This suggests that moderately elevated S100B levels might be beneficial in the context of IFN- $\beta$ effects on $\mathrm{BBB}$ integrity and inhibition of more severe steps of inflammatory 
activity such as T-cell invasion and subsequent neuro-axonal degeneration.

\section{Acknowledgements}

This study was devised as part of a study into biomarkers for neurodegeneration supported by the Multiple Sclerosis Society of Great Britain and Northern Ireland (AP, GG), the BR Kirk Fund of the Institute of Neurology (AP, ET) and the Foundation for Polish Science (KR).

\section{References}

[1] C. Lucchinetti, W. Brück, M. Rodriguez, H. Lassmann, Distinct patterns of multiple sclerosis pathology indicate heterogeneity in pathogenesis, Brain Pathol 6 (1996) 259-274.

[2] A. Bitsch, W. BrA $\frac{1}{4}$ ck, Differentiation of multiple sclerosis subtypes: implications for treatment, CNS Drugs 16 (2002) 405-418.

[3] J. Noseworthy, C. Lucchinetti, M. Rodriguez, B. Weinshenker, Multiple sclerosis, N Engl J Med 343 (2000) 938-952.

[4] A. Petzold, M. Eikelenboom, D. Gveric, G. Keir, M. Chapman, R. Lazeron, et al., Markers for different glial cell responses in multiple sclerosis: Clinical and pathological correlations, Brain 125 (2002) 1462-1473.

[5] G. Giovannoni, S. Heales, N. Silver, J. O'Riordan, R. Miller, J. Land, et al., Raised serum nitrate and nitrite levels in patients with multiple sclerosis, J Neurol Sci 145 (1997) 77-78.

[6] F. Lublin, S. Reingold, Defining the clinical course of multiple sclerosis: results of an international survey National Multiple Sclerosis Society (USA) Advisory Committee on Clinical Trials of New Agents in Multiple Sclerosis, Neurology 46 (1996) 907-911.

[7] A. Petzold, G. Keir, Green, et al., A specific ELISA for measuring neurofilament heavy chain phosphoforms, J Immunol Methods 278 (2003) 179-190.

[8] K. Miranda, M. Espey, D. Wink, A rapid, simple spectrophotometric method for simultaneous detection of nitrate and nitrite, Nitric Oxide 5 (2001) 62-71.

[9] A. Green, G. Keir, E. Thompson, A specific and sensitive ELISA for measuring S-100b in cerebrospinal fluid, J Immunol Meth 205 (1997) 35-41.

[10] G. Keir, N. Tasdemir, E. Thompson, Cerebrospinal-fluid ferritin in brain necrosis - evidence for local synthesis, Clin Chim Acta 216 (1993) 153-166. 
[11] K. Wandinger, C. Sturzebecher, B. Bielekova, G. Detore, A. Rosenwald, L. Staudt, et al., Complex immunomodulatory effects of interferon-beta in multiple sclerosis include the upregulation of $\mathrm{T}$ helper 1-associated marker genes, Ann Neurol 50 (3) (2001) 349-357.

[12] P. Rieckmann, K. Smith, Multiple sclerosis: more than inflammation and demyelination, Trends Neurosci 24 (2001) 435-407.

[13] R. Ransohoff, P. KivisÂAkk, G. Kidd, Three or more routes for leukocyte migration into the central nervous system, Nature Reviews 3 (2003) 569-581.

[14] A. Petzold, K. Rejdak, G. Plant, Axonal degeneration and inflammation in acute optic neuritis, J Neurol Neurosurg Psychiatry 75 (2004) 1178-1780.

[15] R. Donato, S100: a multigenic family of calcium-modulated proteins of the EFhand type with intracellular and extracellular functional roles, Int J Biochem Cell Biol 33 (2001) 637-668. 
Table 1: Demographic characteristics of patients classified according to clinical criteria of treatment failure. The relapse frequency is given in number of relapses per year. The age and disease duration are given in years. Levels of plasma $\mathrm{S100B}, \mathrm{NO}_{x}, \mathrm{NfH}^{\text {SMI35 }}$ median (range) in patients based on clinical criteria of treatment failure. Statistical comparisons are indicated in the following order: control versus non-responder; controls versus responder; non-responder versus responder. NS = not significant.

\begin{tabular}{llllr}
\hline & Control & \multicolumn{2}{c}{ MS patients } & \\
\cline { 3 - 4 } & & Non-responder & Responder & P value \\
\hline Number & 14 & $11(37 \%)$ & $19(63 \%)$ & $<0.05 ;<0.05 ;$ NS \\
Age & $36(24-59)$ & $26(23-42)$ & $33(19-40)$ & NS \\
Sex (F:M) & $8: 6$ & $9: 2$ & $16: 3$ & $<0.001$ \\
Relapse frequency & N/A & $2(2-8)$ & $1(0-1)$ & NS \\
Disease duration & N/A & $8(2-14)$ & $7(1-18)$ & NS \\
EDSS & N/A & $3.5(1.0-6.5)$ & $3.5(1.5-6.5)$ & NS; <0.001; <0.05 \\
IFN 1a n $(\%)$ & N/A & $1(9 \%)$ & $3(16 \%)$ & $0.01 ;$ NS; NS \\
IFN 1b n $(\%)$ & N/A & $10(91 \%)$ & $16(84 \%)$ & $<0.001 ;<0.001 ;$ NS \\
S100B $(\mathrm{pg} / \mathrm{mL})$ & $2(0-11)$ & $11.7(0.1-53.2)$ & $42.9(16.5-483.0)$ & \\
NO $(\mu \mathrm{M})$ & $37(35-39)$ & $42.4(25.2-74.5)$ & $39(17.3-81.0)$ & \\
NfH ${ }^{S M I 35}(\mathrm{pg} / \mathrm{mL})$ & $4.5(0-41)$ & $500(0.1-2,200)$ & $300(0.1-2,400)$ & $<$ \\
\hline
\end{tabular}




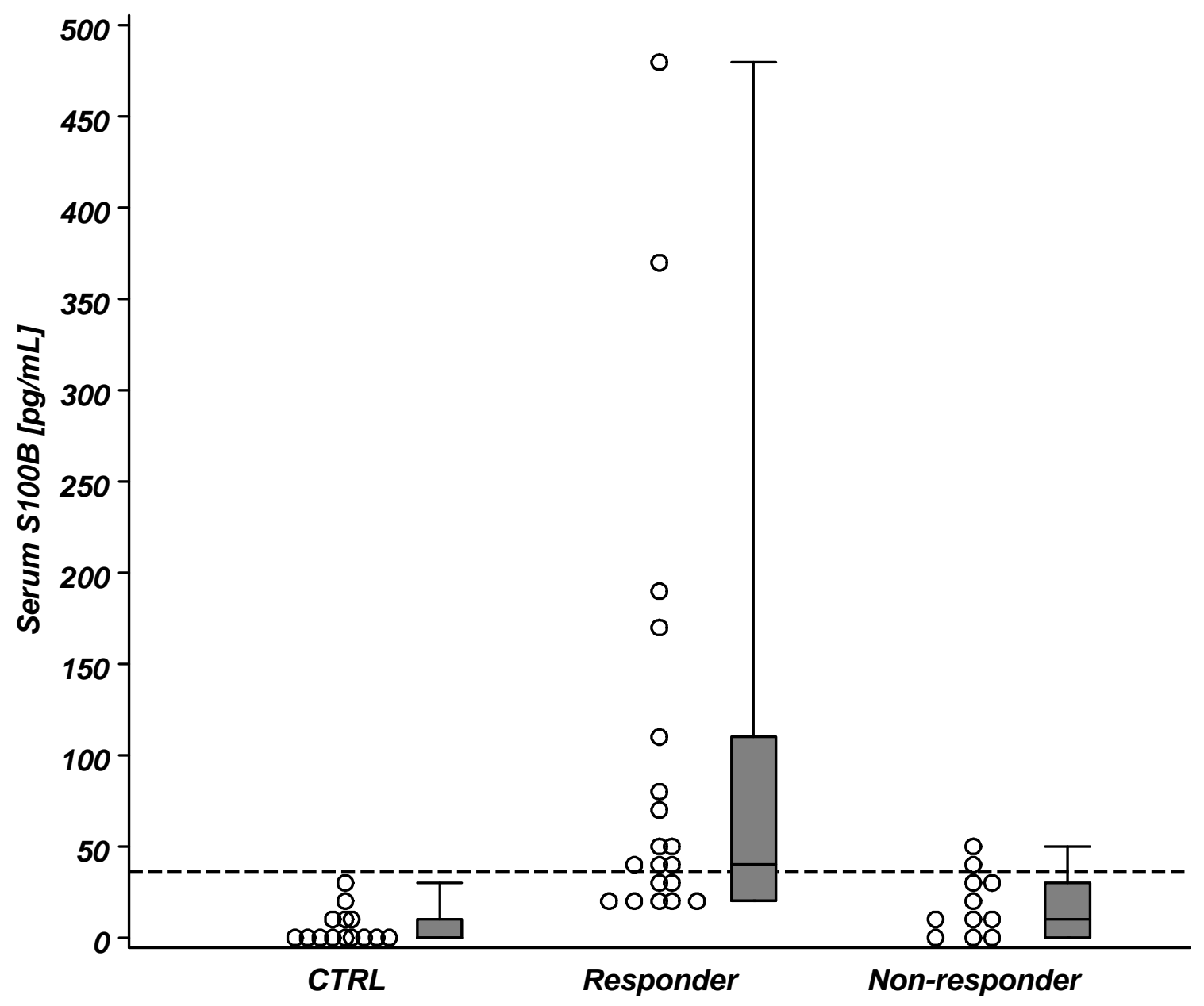

Figure 1. Plasma $S 100 B(\mathrm{pg} / \mathrm{mL})$ levels in normal controls, treatment non-responders and treatment responders. The dotted horizontal line indicates the cutoff of 33 $\mathrm{pg} / \mathrm{mL}$ (100\% cumulative frequency of the control group). 